\title{
Covering Lemmas and BMO Estimates for Eigenfunctions on Riemannian Surfaces
}

Guozhen Lu

\begin{abstract}
The principal aim of this note is to prove a covering Lemma in $\mathbf{R}^{2}$. As an application of this covering lemma, we can prove the BMO estimates for eigenfunctions on two-dimensional Riemannian manifolds $\left(M^{2}, g\right)$. We will get the upper bound estimate for $\|\log \mid u\|_{B M O}$, where $u$ is the solution to $\Delta u+\lambda u=0$, for $\lambda>1$ and $\Delta$ is the Laplacian on $\left(M^{2}, g\right)$. A covering lemma in homogeneous spaces is also obtained in this note.
\end{abstract}

\section{Introduction}

Let $M$ be a smooth, compact and connected Riemannian manifold without boundary. Let $\Delta$ denote the Laplacian on $M$. Assume that $u$ is the solution to $\Delta u+\lambda u=0, \lambda>1$, i.e., $u$ is an eigenfunction with eigenvalue $\lambda$.

Many authors have studied the estimates of the BMO norm and the Nodal sets of eigenfunctions, see $[\mathrm{DF} 1],[\mathrm{DF} 2],[\mathrm{C}],[\mathrm{B}]$, and $[\mathrm{CM}]$. In $[\mathrm{C}]$, Cheng proved that $u$ vanishes at most to order $c \lambda$ in the two-dimensional case. In [B], Brüning showed the lower bound for the volume of the nodal set for $C^{\infty}$ metrics on Riemannian surfaces. Donnelly and Fefferman, see [DF1] and [DF2], obtained the growth property, estimates of the BMO norm and bounds for the volume of the Nodal set of eigenfunctions for all $n \geq 2$. Recently, Chanillo and Muckenhoupt, see [CM], improved the results of [DF2] for $n \geq 3$. 
The purpose of this note is to get a better BMO estimate of eigenfunctions in the two-dimensional case. The main result is the following

Theorem 1. (BMO estimate for $\log |u|$ ) For $u, \lambda$ as above, and for $\epsilon>0$

$$
\|\log |u|\|_{B M O} \leq c \lambda^{15 / 8+\epsilon}
$$

where $c=c(\alpha, M)$ is independent of $\lambda$.

The proof of Theorem 1 is based on the following covering lemma which is of independent interest.

Lemma 1. (Covering Lemma) Let $\delta>0$ be small enough, then given any finite collection of balls $\left\{B_{\alpha}\right\}_{\alpha \in I}$, one can select a subcollection $B_{1}, \ldots, B_{N}$ such that

$$
\bigcup_{\alpha} B_{\alpha} \subset \sum_{i=1}^{N}(1+\delta) B_{i}
$$

and

$$
\sum_{i=1}^{N} \chi_{B_{i}}(x) \leq c \delta^{-7 / 4} \log \frac{1}{\delta}
$$

where $c$ is an constant independent of $\delta$ and the given collection of balls.

The motivation of this note is from $[\mathrm{CM}]$. In $[\mathrm{CM}]$, a covering lemma plays an important role. Lemma 1 is an improvement of the covering lemma for the two-dimensional case in $[\mathrm{CM}]$ so that we can get a better estimate of the BMO norm for eigenfunctions by using Lemma 1 and adapting the proof given in $[\mathrm{CM}]$ for $n \geq 3$. We would like to point out that it is quite possible to prove a covering lemma in case $n \geq 3$ which is better than that in $[\mathrm{CM}]$ by modifying the proof of Lemma 1.

This note is organized as follows: Section 2 explains why we should use a new selection of balls in order to get a better covering lemma than that in $[\mathrm{CM}]$; Section 3 is devoted to the proof of a covering lemma in homogeneous spaces which is of independent interest; Section 4 and 5 deal with the proof of Lemma 1; Section 6 is devoted to the proof of Theorem 1.

One world about notations: Throughout this note, $C$ and $c$ will always denote generic positive constants independent of the given balls $\left\{\boldsymbol{B}_{\alpha}\right\}_{\alpha \in I}$ and $\delta>0 ; \varrho(B)$ will denote the radius of the ball $B ; B(x, r)$ will denote the ball centered at $x$ and of radius $r$.

Acknowledgement. This note represents a part of my $\mathrm{Ph}$. D. Thesis at Rutgers University under the guidance of Prof. S. Chanillo. I am very grateful to my advisor for his constant encouragement and for sharing his ideas and time with me. 


\section{A Covering Lemma due to Chanillo-Muckenhoupt}

In this section, we first recall a covering lemma in $[\mathrm{CM}]$.

Lemma 2.1 (Chanillo-Muckenhoupt) Fix $0<\delta<1 / 2$. Given any finite collection of balls $\left\{B_{\alpha}\right\}_{\alpha \in I}$ in $\mathbf{R}^{n}$, one can select a subcollection $B_{1}, \ldots, B_{N}$ such that

$$
\begin{gathered}
\bigcup_{\alpha} B_{\alpha} \subset \bigcup_{i=1}^{N}(1+\delta) B_{i} \\
\sum_{i=1}^{N} \chi_{B_{i}}(x) \leq 4^{n} \delta^{-n}
\end{gathered}
$$

for all $x \in \mathbf{R}^{n}$.

In the proof of Lemma 2.1, one first selects a ball $B_{1}$ with the largest radius in $\left\{\boldsymbol{B}_{\alpha}\right\}_{\alpha \in \mathrm{I}}$. Having selected $\boldsymbol{B}_{1}, \ldots, \boldsymbol{B}_{k-1}$, one selects $\boldsymbol{B}_{k}$ so that

$$
B_{k} \lessdot \bigcup_{i=1}^{k-1}(1+\delta) B_{i}
$$

and $\boldsymbol{B}_{k}$ has the largest possible radius in the collection $\left\{\boldsymbol{B}_{\alpha}\right\}_{\alpha \in I} \backslash\left\{\boldsymbol{B}_{i}\right\}_{i=1}^{k-1}$. Here we want to point out that (ii) of Lemma 2.1 is the best possible result which can be obtained by the above selection of balls. We show this by giving the following:

EXAMPLE: Consider a family of unit balls centered inside a cube

$$
Q=\{(x, y): 0 \leq x \leq 1 / 2,0 \leq y \leq 1 / 2\} \subset \mathbf{R}^{2}
$$

and the centers of these balls have coordinates

$$
\{(2 k \delta, 4 l \delta)\}, 0 \leq k \leq\left[1 / 4 \delta^{-1}\right], 0 \leq l \leq\left[1 / 8 \delta^{-1}\right]
$$

where $[\cdot]$ denotes the largest integer part. We also denote the unit ball with center $(2 k \delta, 4 l \delta)$ by $B_{k, l}$. We now select the balls in the following order:

$$
\begin{aligned}
& B_{0,0}, B_{1,0}, \ldots, B_{\left|1 / 4 \delta^{-1}\right|, 0}, B_{0,1}, B_{1,1}, \ldots, B_{\left|1 / 4 \delta^{-1}\right|, 1}, \ldots, B_{0,\left|1 / 8 \delta^{-1}\right|}, B_{1,\left|1 / 8 \delta^{-1}\right|}, \ldots, \\
& B_{\left|1 / 4 \delta^{-1}\right|,\left[1 / 8 \delta^{-1}\right]},
\end{aligned}
$$


Then an easy calculation shows (2.2) holds. This selection exactly follows the method used in the proof of Lemma 2.1. But

$$
\sum_{i=1}^{N} \chi_{B_{i}}(x, y) \approx \delta^{-2}, \text { for } 0 \leq x \leq 1 / 2,0 \leq y \leq 1 / 2 \text {. }
$$

Therefore the above example tells us that we need to use a new selection of balls in order to get a better result than that in Lemma 2.1.

\section{A Covering Lemma in Homogeneous Spaces}

In this section, we are going to prove a covering lemma in homogeneous metric spaces with a doubling Borel measure. This section is independent of the others. We will apply the technique of partitioning the radii of balls to reduce the proof of the main lemma to a certain basic case. The proof as given below is an adaptation to homogeneous spaces of the proof on $\mathbf{R}^{n}$ due to $\mathrm{S}$. Chanillo.

We say a pair $(X, \varrho)$ is a homogeneous metric space in the sense of Coifman and Weiss, if the following hold:

(i) $\varrho: X \times X \rightarrow \mathbf{R}^{+}$satisfies the following conditions:

$$
\begin{gathered}
\varrho(x, y)=0 \text { if and only if } x=y \\
\varrho(x, y)=\varrho(y, x) \\
\varrho(x, y) \leq K[\varrho(x, z)+\varrho(z, y)]
\end{gathered}
$$

where $K$ is a constant independent of $x, y, z$ and

(ii) there is a Borel measure $\mu$ such that

$$
0<\mu(B(x, r)) \leq A \mu(B(x, r / 2))<+\infty,
$$

where $A$ is a constant independent of the ball $B(x, r)$ centered at $x$ and with radius $r$.

An easy consequence of (3.1) is

$$
\mu(B(x, r)) \leq A^{\log _{2}\left(r / r^{\prime}\right)+1} \mu\left(B\left(x, r^{\prime}\right)\right)
$$

for any $x \in X$ and $0<r^{\prime}<r$. 
According to [CoW], both Vitali type and Whitney type covering lemmas are true. It is well-known that Besicovitch covering lemma may not be true in homogeneous spaces as pointed out in [SW]. Sometimes a Vitali covering lemma is not good enough for applications as in [CM], but the following covering lemma could be a replacement of both Vitali and Besicovitch covering lemmas.

Lemma 3.3. Let $\delta>0$ be small enough and $\left\{B_{\alpha}\right\}_{\alpha \in I}$ be a finite collection of balls in $X$. If there exists a doubling Borel measure $\mu$ on $X$ satisfying (3.1), then one can select a subcollection $B_{1}, \ldots, B_{N}$ such that

$$
\begin{aligned}
& \bigcup_{\alpha} B_{\alpha} \subset \bigcup_{i=1}^{N}(K+\delta) B_{i} \\
& \sum_{i=1}^{N} \chi_{B_{i}}(x) \leq C \delta^{-d} \log \frac{1}{\delta}
\end{aligned}
$$

where $C$ depends only on $K$ and $A$, and $d=\log _{2} A$.

Proof. Select a ball $\boldsymbol{B}$ with the largest radius in $\left\{\boldsymbol{B}_{\alpha}\right\}$. Having selected balls $B_{1}, \ldots, B_{k-1}$, select $B_{k}$ such that

$$
B_{k} \nsubseteq \bigcup_{i=1}^{k-1}(K+\delta) B_{i}
$$

and $B_{k}$ has the largest radius out of the collection

$$
\left\{\boldsymbol{B}_{\alpha}\right\}_{\alpha \in \mathrm{I}} \backslash\left\{\boldsymbol{B}_{i}\right\}_{i=1}^{k-1}
$$

The subcollection $B_{1}, \ldots, B_{M}$ chosen by the above selection obviously satisfies

$$
\bigcup_{\alpha} B_{\alpha} \subset \bigcup_{i=1}^{M}(K+\delta) B_{i}
$$

Now we prove (3.5) in the lemma. We first fix any point $x_{0} \in X$. With no loss of generality, we may assume $x_{0} \in \bigcap_{i=1}^{l} B_{i}$. We also assume $B_{i}=B_{i}\left(z_{i}, r_{i}\right)$. By the selection of $\left\{B_{\alpha}\right\}_{i=1}^{M}$, we know $r_{l} \leq r_{l-1} \leq \ldots \leq r_{1}$. We note that there exists $\sigma=\sigma\left(x_{0}\right)$ such that 


$$
\sum_{i=1}^{l} \chi_{B_{i}}\left(x_{0}\right)=\sum_{k=1}^{\sigma} \sum_{2^{k} r_{l} \leq r_{i}<2^{k+1} r_{l}} \chi_{B_{i}}\left(x_{0}\right)
$$

We now have the following claims.

Claim (1): $\delta 2^{\sigma} \leq 2 K^{2}$

If not, then $\delta r_{1} \geq \delta 2^{\sigma} r_{l}>2 K^{2} r_{l}$.

We note that for $y \in B_{l}=B\left(z_{l}, r_{l}\right)$,

$$
\begin{aligned}
\varrho\left(y, z_{1}\right) & \leq K\left[\varrho\left(y, x_{0}\right)+\varrho\left(x_{0}, z_{1}\right)\right] \\
& \leq K\left\{K\left[\varrho\left(y, z_{l}\right)+\varrho\left(z_{l}, x_{0}\right)\right]+\varrho\left(x_{0}, z_{1}\right)\right\} \\
& \leq K\left[2 K r_{l}+r_{1}\right]=2 K^{2} r_{l}+K r_{1} \\
& <\delta r_{1}+K r_{1} \\
& =(K+\delta) r_{1}
\end{aligned}
$$

Thus $B_{l} \subset(K+\delta) B_{1}$ which is a contradiction to (3.6). Thus claim (1) holds, i.e., $\sigma \leq C \log \frac{1}{\delta}$, where $C$ only depends on $K$.

Claim (2): For the subcollection $\left\{B_{i j}\right\}_{j=1}^{N_{k}}$ of $\left\{B_{i}\right\}_{i=1}^{l}$ with $2^{k} r_{l} \leq \varrho\left(B_{i j}\right)<2^{k+1} r_{l}$, we have

$$
\varrho\left(z_{i j}, z_{i h}\right)>\frac{2^{k} r_{l} \delta}{K} \text { for } j \neq h, 1 \leq j, h \leq N_{k}
$$

For simplicity, we drop the subscripts and denote $B_{i_{j}}$ and $B_{i_{h}}$ by $B_{i}$ and $B_{h}$ respectively. We also assume $j>h$. If the claim were not true, we would have for $y \in B_{j}=B\left(z_{j}, r_{j}\right)$

$$
\begin{aligned}
\varrho\left(y, z_{h}\right) & \leq K\left[\varrho\left(y, z_{j}\right)+\varrho\left(z_{j}, z_{h}\right)\right] \\
& \leq K\left[r_{j}+\frac{2^{k} \delta}{K}\right] \\
& \leq K r_{j}+2^{k} r_{l} \delta \leq K r_{j}+\delta r_{j} \\
& =(K+\delta) r_{j} \\
& \leq(K+\delta) r_{h} .
\end{aligned}
$$

This implies $(K+\delta) B_{h} \supset B_{j}$ which is again a contradiction to (3.6).

Claim (3): The balls $\left\{B\left(z_{j}, \frac{2^{k}}{2 K^{2}}\right)\right\}_{j=1}^{N_{k}}$ are mutually disjoint. 
We set $R_{1}=2^{k} r_{l} \delta / 2 K^{2}$. If the claim were not true, there would exist $y \in B\left(z_{j}, R_{1}\right) \cap\left(z_{h}, R_{1}\right)$ for some $j \neq h$. Thus

$$
\left.\varrho\left(z_{j}, z_{h}\right)\right] \leq K\left[\varrho\left(z_{j}, y\right)+\varrho\left(y, z_{h}\right) \leq\left[\frac{2^{k} r_{l} \delta}{2 K^{2}}+\frac{2^{k} r_{l} \delta}{2 K^{2}}\right]=\frac{2^{k} r_{l} \delta}{K},\right.
$$

which is a contradiction to the claim (2).

Claim (4): The balls $\left\{B_{j}\right\}_{j=1}^{N_{k}}$ are all contained in $B\left(x_{0}, R_{2}\right)$, where $R_{2}=K 2^{k+2} r_{l}$.

For $y \in B\left(z_{j}, r_{j}\right)$, we have

$$
\begin{aligned}
\varrho\left(y, x_{0}\right) & \leq K\left[\varrho\left(y, z_{j}\right)+\varrho\left(z_{j}, x_{0}\right)\right] . \\
& \leq 2 K r_{j} \leq 2 K \cdot 2^{k+1} \mathrm{r}_{l} \\
& =K 2^{k+2} r_{l} .
\end{aligned}
$$

Thus the claim holds.

Claim (5): $B\left(x_{0}, R_{2}\right) \subset B\left(z_{j}, R_{3}\right)$ for each $1 \leq j \leq N_{k}$, where $R_{3}=$ $K(2 K+1) 2^{k+1} r_{l}$.

In fact, for $y \in B\left(x_{0}, R_{2}\right)$, we have

$$
\begin{aligned}
\varrho\left(y, z_{j}\right) & \leq K\left[\varrho\left(y, x_{0}\right)+\varrho\left(x_{0}, z_{j}\right)\right] \\
& \leq K\left[R_{2}+r_{j}\right] \leq K\left[2^{k+2} r_{l} k+2^{k+1} r_{l}\right] \\
& =K(2 K+1) 2^{k+1} r_{l}=R_{3} .
\end{aligned}
$$

This proves claim (5).

Now by claims (3) and (4), we have

$$
\sum_{j=1}^{N_{k}} \mu\left(B\left(z_{j}, R_{1}\right)\right) \leq \mu\left(B\left(x_{0}, R_{2}\right)\right)
$$

and by claim (5) and (3.2), we have

$$
\mu\left(B\left(x_{0}, R_{2}\right)\right) \leq \mu\left(B\left(z_{j}, R_{3}\right)\right) \leq A^{\log _{2}\left(R_{3} / R_{1}\right)+1} \mu\left(B\left(z_{j}, R_{1}\right)\right) .
$$

Therefore, from (3.8) and (3.9), it is easy to see

$$
N_{k} \leq A^{\log _{2}\left(R_{3} / R_{2}\right)+1}=A^{\left.\log _{2} \mid 4 K^{3}(2 K+1) / \hat{b}\right]} .
$$


By using $A=2^{d}$ and an easy calculation, we get

$$
N_{k} \leq\left[8 K^{3}(2 K+1)\right]^{d} \delta^{-d}
$$

Now,

$$
\begin{aligned}
\sum_{i=1}^{l} \chi_{B_{i}}\left(x_{0}\right) & =\sum_{k=0}^{\sigma} \sum_{2^{k} r_{r} \leq r_{j} \leq 2^{k+1} r_{l}} \chi_{B_{j}}\left(x_{0}\right) \\
& \leq \sum_{k=0}^{\sigma} N_{k} \leq \sum_{k=1}^{\sigma}\left[8 K^{3}(2 K+1)\right]^{d} \delta^{-d} \\
& \leq C\left(\log \frac{1}{\delta}\right) \delta^{-d}
\end{aligned}
$$

where $C$ only depends on $K$ and $A$. This shows (3.5) and thus completes the proof of lemma (3.3).

\section{A Basic Covering Lemma}

The purpose of this section is to prove a covering lemma for balls which centered in any given cube in $R^{2}$ with sidelenght $\sqrt{\delta}$. Moreover, these balls have radii whose values are close to one another.

Lemma 4.1. Let $\delta>0$ be given small enough. Given any cube $Q$ in $\mathbf{R}^{2}$ with sidelength $\sqrt{\delta}$ and given any finite collection of balls $\left\{B_{\alpha}\right\}_{\alpha \in \mathrm{I}}$ with $r \leq \varrho\left(B_{\alpha}\right) \leq$ $\leq r+\delta$, for some $1 \leq r \leq 2$ and centered in this cube $Q$, one can select a subcollection of balls $B_{1}, \ldots, B_{N}$ such that

$$
\bigcup_{\alpha} B_{\alpha} \subset \bigcup_{i=1}^{N}(1+c \delta) B_{i}
$$

$$
N \leq c \delta^{-1 / 4}
$$

where $c$ is an absolute constant independent of $\delta$ and the given balls.

In order to prove Lemma 4.1, we need the following propositions.

Proposition 4.1. The sum of all exterior angles of any convex polygon is $2 \pi$.

This a well-known formula in plane geometry. One may also deduce this fact from the Gauss-Bonnet formula in differential geometry. 
Proposition 4.2. The perimeter of any convex plane polygon contained inside a cube $Q$ with sidelength $\sqrt{\delta}$ is less than $2 \pi \sqrt{2 \delta}$.

The proof of the above Proposition 4.2 uses the Cauchy-Crofton formula in $\mathbf{R}^{2}$. It states that for a given regular plance curve $C$ with length $l$, the measure of the set of straight lines (counted with multiplicities) which meet $C$ is equal to $2 l$. A proof of this assertion may be found on page 41 in [Do]. A higher dimentional version of Cauchy-Crofton formula is proved in $[\mathrm{F}]$.

PROOF OF PROPOSITION 4.2. Let $\partial P$ be the boundary of the convex polygon $P$ inside $Q$, and let $S$ be the set of straight lines which meet $P$. Then if we denote by $\varrho$ the distance from the origin to the lines and by $\theta$ the angle between the positive $x$-axis and the line, and assume without loss of generality,

$$
O=\{(x, y): 0 \leq x \leq \sqrt{\delta}, 0 \leq y \leq \sqrt{\delta}\},
$$

we have

$$
2 \text { length }(\partial P)=\int_{S} d \pi d \theta \leq \int_{0}^{2 \pi} \int_{0}^{\sqrt{2} \delta} 2 d \varrho d \theta=4 \pi \sqrt{2 \delta}
$$

Thus, length $(\partial P) \leq 2 \pi \sqrt{2 \delta}$.

Remark: After this note was prepared, Prof. B. Muckenhoupt pointed out that the proof of Proposition 4.2 can be simplified by projecting $\partial P$ to $\partial Q$.

Proposition 4.3. Given any $\delta>0$ small enough and oriented rectangle $R$ in $\mathbf{R}^{2}$ with sidelength $\sqrt{\delta}$ and $\delta$. Let $\left\{B_{\alpha}\right\}_{\alpha \in \mathrm{I}}$ be a finite collection of balls centered in $R$ and with radii $r \leq \varrho\left(B_{\alpha}\right) \leq r+\delta$ for some $1 \leq r \leq 2$. Then we only need to select at most two balls $B_{1}$ and $B_{2}$ such that

$$
\bigcup_{\alpha} B_{\alpha} \subset \bigcup_{i=1}^{2}(1+c \delta) B_{i}
$$

PROOF. If there are no more than two balls in the given collection, then there is nothing to prove. If there are more than two centers in $R$, then we select two balls $B_{1}$ and $B_{2}$ with centers $O_{1}=\left(x_{o_{1}}, y_{o_{1}}\right)$ and $O_{2}=\left(x_{o_{2}}, y_{o_{2}}\right)$ respectively such that one of the centers is on the extreme left, the other is on the extreme right (as shown in Figure 1). We claim

$$
\bigcup_{\alpha} B_{\alpha} \leq \bigcup_{i=1}^{2}(1+c \delta) B_{i}
$$


We will assume with no loss of generality that both $B_{1}$ and $B_{2}$ have the smallest possible radius in our collection, that is $r$. We may also assume with no loss of generality that $y_{o_{1}}=y_{o_{2}}$, then the ball $B_{1}^{\prime}$ with radius $r$ and center $\left(x_{o_{1}}, y_{o_{2}}\right)$ is contained in $(1+2 \delta) B_{1}$ and thus if we prove our claim with $B_{1}$ replaced by $B_{1}^{\prime}$, our proposition will be proved.

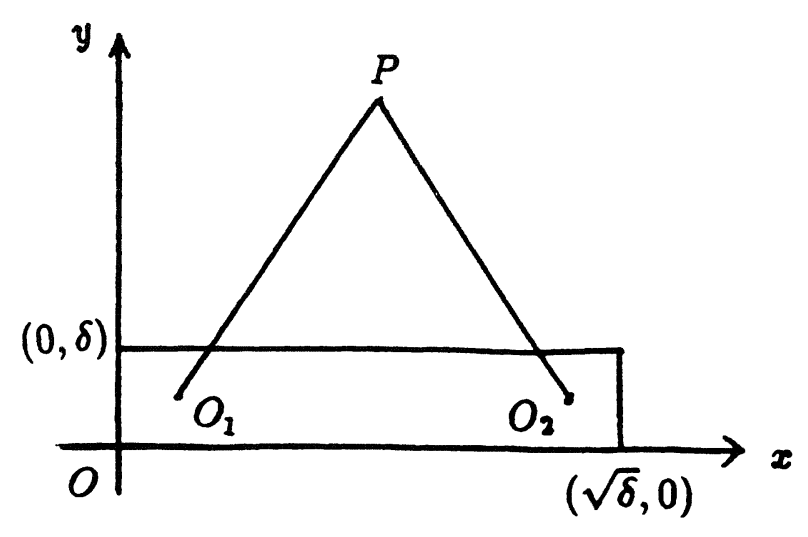

Figure 1

Let $P$ denote the intersection point of $(1+c \delta) B_{1}$ and $(1+c \delta) B_{2}$. We now show that dist $(P, \partial R)>r+2 \delta$. This will prove our claim. Using the fact that $y_{o_{1}}=y_{o_{2}}$, we see that

$$
\begin{aligned}
\operatorname{dist}(P, \partial R) & \geq \operatorname{dist}\left(P, \overline{O O_{2}}\right)-\delta \\
& \geq\left[(1+c \delta)^{2} r^{2}-(\sqrt{\delta} / 2)^{2}\right]^{1 / 2}-\delta .
\end{aligned}
$$

By choosing $c \geq 4$, we get

$$
\left[(1+c \delta)^{2} r^{2}-\delta / 4\right]^{1 / 2}-\delta>r+2 \delta
$$

since $r \geq 1$. This proves our claim and the proposition.

We are also going to need the following:

Proposition 4.4. Let $O_{1} O_{2} O_{3}$ be a triangle with sidelength less than $c \sqrt{\delta}$. Suppose $\left\{B_{i}\right\}_{i=1}^{3}$ are three balls centered at $\left\{O_{i}\right\}_{i=1}^{3}$ and with $r \leq \varrho\left(B_{i}\right) \leq r+\delta$ for some $1 \leq r \leq 2$. Then any ball $B$ with $r \leq \varrho(B) \leq r+\delta$ and centered at some $O$ inside the triangle $O_{1} O_{2} O_{3}$ can be covered by $\cup_{i=1}^{3}(1+c \delta) B_{i}$ for some absolute constant $c$. 
PROOF.

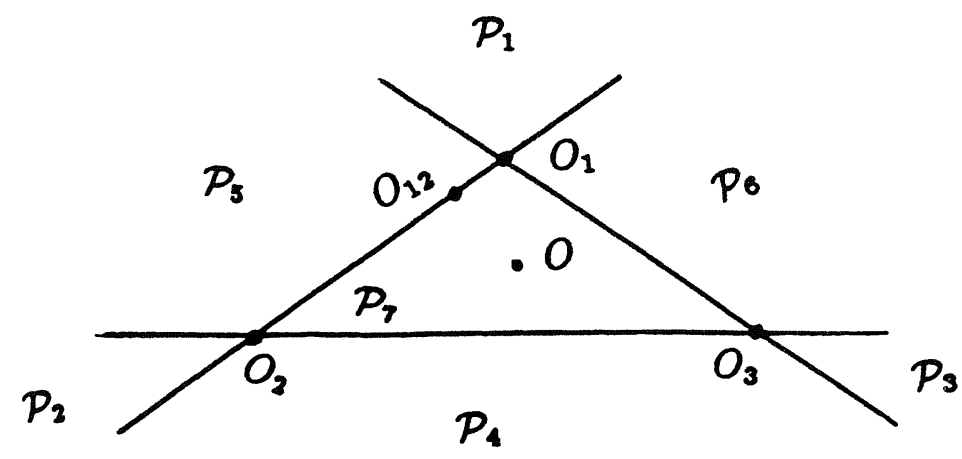

Figure 2

As shown in Fig. 2, we extend segments $\mathrm{O}_{1} \mathrm{O}_{2}, \mathrm{O}_{2} \mathrm{O}_{3}, \mathrm{O}_{3} \mathrm{O}_{1}$ to lines. Then these three lines subdivide $\mathbf{R}^{2}$ into seven pieces $\left\{P_{i}\right\}_{i=1}^{7}$. Obviously, $B \cap P_{1}$, $B \cap P_{2}$ and $B \cap P_{3}$ can be covered by $(1+c \delta) B_{1},(1+c \delta) B_{2}$ and $(1+c \delta) B_{3}$ respectively. Now let $O_{12}$ denote the point $O_{1} O_{2}$ nearest to $O$ and let $B^{\prime}$ be the ball centered at $O_{12}$ of radius $r+\delta$. Then $B^{\prime} \cap P_{5} \supset B \cap P_{5}$ by the above choice of $O_{12}$. But by Proposition 4.3, $(1+c \delta) B_{1} \cup$ $(1+c \delta) B_{2} \supset B^{\prime}$. Thus

$$
B \cap P_{5} \subset \bigcup_{i=1}^{3}(1+c \delta) B_{i}
$$

A similar argument shows that

$$
B \cap P_{j} \subset \bigcap_{i=1}^{3}(1+c \delta) B_{i} \text { for } j=4,6 .
$$

This completes the proof of the proposition.

Proposition 4.5. Let $\left\{B_{\alpha}\right\}_{\alpha \in \mathrm{I}}$ be a finite collection of balls with $r \leq \varrho\left(B_{\alpha}\right)$ $\leq r+\delta$ for some $1 \leq r \leq 2$. If there exists a subcollection $\left\{B_{i}\right\}_{i=1}^{m}$ of $\left\{B_{\alpha}\right\}$ and another further subcollection $\left\{B_{i_{k}}\right\}_{k=1}^{n}$ of $\left\{B_{i}\right\}$ such that

$$
\bigcup_{\alpha} B_{\alpha} \subset \bigcup_{i=1}^{m}(1+c \delta) B_{i}
$$




$$
B_{i} \subset \bigcup_{k=1}^{n}(1+c \delta) B_{i_{k}}
$$

for each $i$. Then

$$
\bigcup_{\alpha} B_{\alpha} \subset \bigcup_{k=1}^{n}\left(1+c^{\prime} \delta\right) B_{i_{k}}
$$

for some $c^{\prime}>c$ independent of $\delta$ and the given balls.

ProOF. It suffices to prove

$$
(1+c \delta) B_{i} \subset \bigcup_{k=1}^{n}\left(1+c^{\prime} \delta\right) B_{i_{k}}
$$

for each $1 \leq i \leq m$. Now fix $i$, let $B_{i}^{\prime}=(1+c \delta) B_{i}, O_{i}$ be the center of $B_{i}$, $\partial B_{i}$ be the boundary of $B_{i}$. Then it is enough to show

$$
B_{i}^{\prime} \backslash B_{i} \subset \bigcup_{i=1}^{n}\left(1+c^{\prime} \delta\right) B_{i_{k}} .
$$

Let $P \subset \partial B_{i}$, we denote by $Q$ the intersection point between $B_{i}^{\prime}$ and the half line starting with the point $O_{i}$ and passing through $P$. Then the length of the segment $P Q$ is

$$
(1+c \delta) \varrho\left(B_{i}\right)-\varrho\left(B_{i}\right)=c \delta \varrho\left(B_{i}\right) \leq 3 c \delta .
$$

Assume $P \in(1+c \delta) B_{i_{k}}$. In fact, dist $\left(P, O_{i_{k}}\right) \leq(1+c \delta) \varrho\left(B_{i_{k}}\right)$, and then for any $z \in P Q$, we have

$$
\begin{aligned}
\operatorname{dist}\left(z, O_{i_{k}}\right) & \leq \operatorname{dist}(z, P)+\operatorname{dist}\left(P, O_{i_{k}}\right) \\
& \leq 3 c \delta+(1+c \delta) \varrho\left(B_{i_{k}}\right) \\
& \leq\left(1+c^{\prime} \delta\right) \varrho\left(B_{i_{k}}\right) .
\end{aligned}
$$

The last inequality follows from $\varrho\left(B_{i_{k}}\right) \geq 1$. Thus the claim follows. We move $P$ along $\partial B_{i}$ and note that the union of all such segments $P Q$ cover $B_{i}^{\prime} \backslash B_{i}$, this shows that

$$
B_{i}^{\prime} \backslash B_{i} \subset \bigcup_{k=1}^{n}\left(1+c^{\prime} \delta\right) B_{i_{k}}
$$


For the remainder of this section, all balls that we will consider have radii $\varrho$ such that $r_{0} \leq \varrho \leq r_{0}+\delta$ for a fixed $r_{0}, 1 \leq r_{0} \leq 2$.

We now begin the proof of Lemma 4.1.

Let $S$ be the collection of centers of the balls $\left\{B_{\alpha}\right\}_{\alpha \in \mathrm{I}}$. Let $V$ be the convex hull of $S$. Then the boundary of $V$ must be a convex plane polygon and the vertices of $V$ consist of centers, say, $O_{1}, O_{2}, \ldots, O_{M}$, enumerated in a clockwise order.

Let us consider any three vertices of $V$, say, $O_{i}, O_{j}, O_{k},(1 \leq i<j<k$ $\leq M)$. We introduce the following:

Definition. If $O_{i} O_{k} \leq \delta^{3 / 4}$, we say the triple $O_{i} O_{j} O_{k}$ is of type I. Otherwise, the triple $O_{i} O_{j} O_{k}$ is of type II. We further split the type II triples into two cases: If $O_{j} A_{i j k} \geq \delta$, we say the triple $O_{i} O_{j} O_{k}$ is of type $\mathrm{II}_{1}$, otherwise, of type $\mathrm{II}_{2}$ (see Figure 3 below).
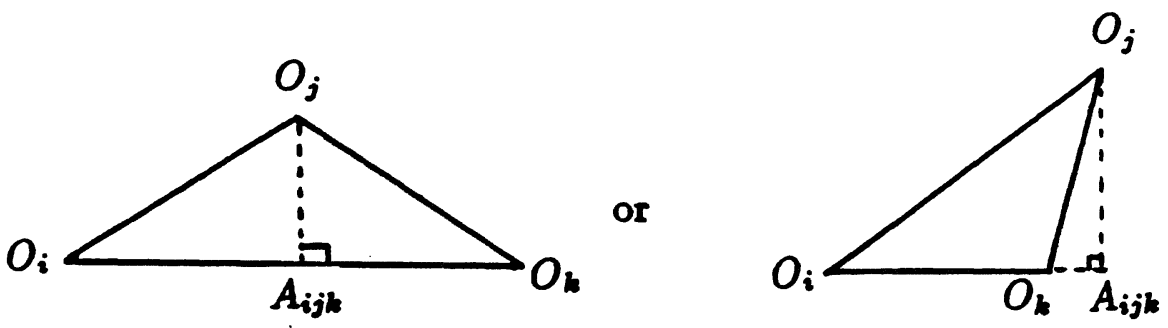

Figure 3

Now we select the vertices $\left\{O_{i_{k}}\right\}$. The balls $\left\{B_{i_{k}}\right\}$ with centers at $\left\{O_{i_{k}}\right\}$ will be the subcollection that shall be used to prove our lemma.

We first make the following observation:

Proposition 4.6. If for some $k \geq 3$, every triple $O_{1} O_{i} O_{k}$ is of type $\mathrm{II}_{2}$ for $2 \leq i \leq k-1$, then all balls centered inside the polygon with vertices $\left\{O_{i}\right\}_{i=1}^{k}$ can be covered by $(1+c \delta) B_{i_{1}} \cup(1+c \delta) B_{i_{2}}$ for some $1 \leq i_{1} \leq i_{2} \leq k$.

PROoF. The polygon $W$ whose vertices are $\left\{O_{i}\right\}_{i=1}^{k}$ is contained inside a rectangle $R$ with sidelengths $\sqrt{2 \delta}$ and $\delta$ and $O_{1} O_{k}$ is a part of one side with length $\sqrt{2 \delta}$ of the rectangle $R$. Then by applying Proposition 4.3, we see that $\cup_{i=1}^{k} B_{i} \subset \cup_{m=1}^{2}(1+c \delta) B_{i_{m}}$. Now any ball $B$ centered in $W$ has its center in a triangle formed by some three vertices of $W$. By Proposition 4.4, $B \subset \cup_{i=1}^{k}(1+c \delta) B_{i}$, thus by Proposition 4.6, we have $B \subset \cup_{m=1}^{2}\left(1+c^{\prime} \delta\right) B_{i_{m}}$. 
We now give the selection procedure. We start with the triple $O_{1} O_{2} O_{3}$. Case 1: If the triple $O_{1} O_{2} O_{3}$ is of type $\mathrm{I}_{\text {or }} \mathrm{II}_{1}$, then we select vertices $O_{1}$, $\mathrm{O}_{2}$ and $\mathrm{O}_{3}$ and pass to the next triple, $\mathrm{O}_{4} \mathrm{O}_{5} \mathrm{O}_{6}$.

Case 2: If the triple $O_{1} O_{2} O_{3}$ is of type $\mathrm{II}_{2}$, then either

(i) Every triple $O_{1} O_{i} O_{M}$ is of type $I I_{2}$ for $2 \leq i \leq M-1$.

Then by Proposition 4.6, We can select $B_{i_{1}}$ and $B_{i_{2}}$ as our subcollection and the proof of Lemma 4.1 is complete in this case. Or

(ii) There exists some $k, 3 \leq k \leq M-1$ such that every triple $O_{1} O_{i} O_{k}$ is of type $\mathrm{II}_{2}$ for $2 \leq i \leq k-1$, but there is some triple $O_{1} O_{i_{0}} O_{k+1}\left(2 \leq i_{0} \leq k\right)$ which is not of type $\mathrm{II}_{2}$, i.e., of type $\mathrm{I}_{\text {or }} \mathrm{II}_{1}$.

Then by Proposition 4.6 again, there are two vertices $O_{i_{1}}$ and $O_{i_{2}}$ for $1 \leq i_{1}, i_{2} \leq k$ such that all balls centered inside the polygon with vertices $\left\{O_{i}\right\}_{i=1}^{k}$ can be covered by $(1+c \delta) B_{i_{1}} \cup(1+c \delta) B_{i_{2}}$. Thus, we select $O_{1}, O_{i_{1}}, O_{i_{2}}$, $O_{k}$ and $O_{k+1}$ in this case (We note that some overlap may occur among the above five vertices since $O_{i_{1}}, O_{i_{2}}$ may be $O_{1}, O_{k}$ ). We then pass to the next triple $O_{k+1} O_{k+2} O_{k+3}$. We continue this selection as before with $O_{k+1}$ playing the role of $O_{1}$. Because there are only finite vertices, this process stops when $O_{1}$ appears again in a new triple. We thus arrive a subcollection of vertices $O_{i_{1}}, O_{i_{2}}, \ldots, O_{i_{L}}$ and a new polygon $Z$ whose vertices are formed by $O_{i_{1}}$, $O_{i_{2}}, \ldots, O_{i_{L}}$.

We now claim that

(i) $Z$ is a convex polygon.

(ii) Any ball $B_{\alpha}$ in the original collection $\left\{\boldsymbol{B}_{\alpha}\right\}_{\alpha \in \mathrm{I}}$ is contained in $\cup_{k=1}^{L}(1+c \delta) B_{i_{k}}$.

By noticing that $Z$ is the intersection of the convex polygon $V$ and the half spaces formed by the lines $O_{i_{k}} O_{i_{k+1}}, 1 \leq k \leq L-1$, and the intersection of convex regions is convex, this show that $Z$ is convex.

To prove (ii), we note that each center $O_{\alpha}$ of the balls $B_{\alpha}$ is in $V$ since $V$ is the convex hull of $S$. Furthermore, recalling that $\left\{O_{i}\right\}$ denotes the vertices of $V$, we see that the center $O_{\alpha}$ must be in one of the triangles $O_{1} O_{m} O_{m+1}(2 \leq m \leq M-1)$. Thus by Propositions 4.4, $B_{\alpha}$ is contained in $\cup_{i=1}^{M}(1+c \delta) B_{i}$. But as abserved above by the selection procedure, $B_{i} \subset \cup_{k=1}^{L} B_{i_{k}}$. Thus $B_{\alpha} \subset \cup_{k=1}^{L}(1+c \delta) B_{i_{k}}$, for all $\alpha \in I$, by Proposition 4.5.

In order to complete the proof of Lemma 4.1, we need the following proposition.

Proposition 4.7. Suppose $\left\{\left(O_{i_{m}}, O_{i_{m}+1}, O_{i_{m}+2}\right)\right\}_{m=1}^{N}$ be the family of type I triples selected from $O_{1}, O_{2}, \ldots, O_{M}$ above, then $N \leq c \delta^{-1 / 4}$. 
ProOF. Since $\overline{O_{i_{m}} O_{i_{m}+2}} \geq \delta^{3 / 4}$ by definition of type I triple. It follows by considering the perimeter of the triangle $O_{i_{m}} O_{i_{m}+1} O_{i_{m}+2}$, that

$$
\overline{O_{i_{m}} O_{i_{m}+1}}+\overline{O_{i_{m}+1} O_{i_{m}+2}} \geq \overline{O_{i_{m}} O_{i_{m}+2}} \geq \delta^{3 / 4} \text {. }
$$

Summing over $m$, we get

$$
\begin{aligned}
N \delta^{3 / 4} & \leq \sum_{m=1}^{N}\left(\overline{O_{i_{m}}} \overline{O_{i_{m}+1}}+\overline{O_{i_{m}} O_{i_{m}+2}}\right) \\
& \leq \text { perimeter of the polygon } Z
\end{aligned}
$$

Since $Z$ is convex and contained in the cube $Q$, the perimeter of $Z$ is no more than $c \sqrt{\delta}$ by Proposition 4.2. Thus $N \delta^{3 / 4} \leq c \sqrt{\delta}$ and consequently $N \leq c \delta^{-1 / 4}$.

Proposition 4.8. Suppose $\left\{\left(O_{i_{m}}, O_{i_{m}+1}, O_{i_{m}+2}\right)\right\}_{m=1}^{N}$ be the family of type $\mathrm{II}_{1}$ triples selected from $O_{1}, O_{2}, \ldots, O_{M}$ above, then $N \leq c \delta^{-1 / 4}$.

ProfF. Given a type $\mathrm{II}_{1}$ triple $\left(O_{i_{m}}, O_{i_{m}+1}, O_{i_{m}+2}\right)$, one has $\overline{O_{i_{m}} O_{i_{m}+2}} \leq \delta^{3 / 4}$ and $\overline{O_{i_{m}+1} A_{m}} \geq \delta$ by the definition of type $\mathrm{II}_{1}$ triple (see Figure 4).

With no loss of generality, we consider those triples such that $\theta_{m} \leq \pi / 4$ since the number of $m$ such that $\theta_{m}>\pi / 4$ is no more than $\frac{2 \pi}{\pi / 4}=8$ by Proposition 4.1. We will show that the exterior angle $\theta_{m}$ is always bounded below by $\delta^{1 / 4} / 2$ for those $\theta_{m} \leq \pi / 4$.

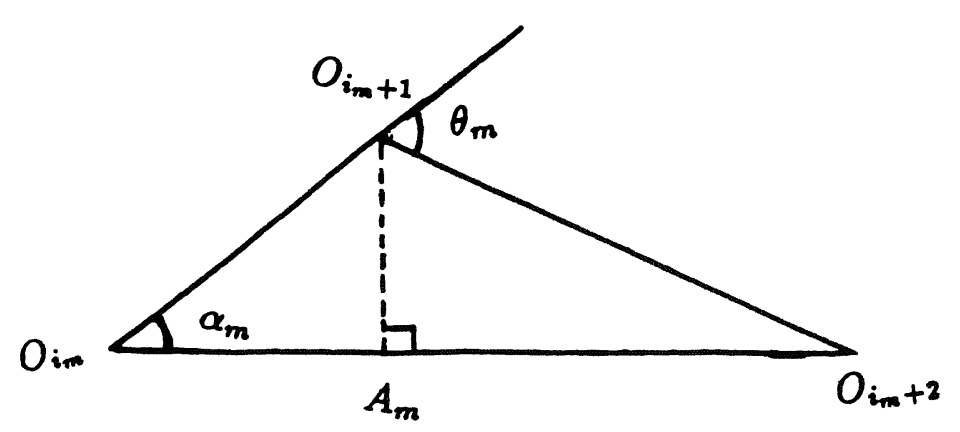

Figure 4 
In fact, we note that for $\theta_{m} \leq \pi / 4$, we have $\tan \theta_{m} \leq 2 \theta_{m}$, thus for $\theta_{m} \leq \pi / 4$, we get

$$
\begin{aligned}
\theta_{m} \geq \alpha_{m} & \geq \frac{1}{2} \tan \alpha_{m}=\frac{1}{2} \frac{\overline{O_{i_{m}+1} A_{m}}}{\overline{O_{i_{m}} A_{m}}} \\
& \geq 1 / 2 \frac{\overline{O_{i_{m}+1} A_{m}}}{\overline{O_{i_{m}} O_{i_{m}+2}}} \geq 1 / 2 \frac{\delta}{\delta^{3 / 4}} \\
& =\frac{1}{2} \delta^{1 / 4} .
\end{aligned}
$$

Applying Proposition 4.1 again, $\sum_{m=1}^{N} \theta_{m} \leq 2 \pi$, where the sum is being taken over the exterior angles which arise in type $\mathrm{II}_{1}$ triples. But $\theta_{m} \geq 1 / 2 \delta^{1 / 4}$, thus $N \leq c \delta^{-1 / 4}$.

Q.E.D.

Finally, we note that the selected vertices $O_{i_{1}}, \ldots, O_{i_{L}}$ are from type I, type $\mathrm{II}_{1}$ or type $\mathrm{II}_{2}$ triples. In the convex polygon $Z$, we consider all maximal chains, where a maximal chain is a union of successive sides of the polygon $Z$ which come from type $\mathrm{I}$ or type $\mathrm{II}_{1}$ triples. Then by the selection procedure, between any two maximal chains, there are no more than five vertices which are probably from type $\mathrm{II}_{2}$ (see case 2 (ii) at the beginning of the selection procedure in this section). Thus the number of these type $\mathrm{II}_{2}$ vertices are less than $c \delta^{-1 / 4}$ also. Therefore, the number of all vertices $O_{i_{1}}, \ldots, O_{i_{1}}$ is less than $c \delta^{-1 / 4}$, and this shows (ii) of Lemma 4.1.

We end this section with the following example which shows (ii) in Lemma 4.1 is the best possible result in each cube with sidelength $\sqrt{\delta}$.

EXAMPLE. Assume that the centers of the unit balls $\left\{\boldsymbol{B}_{\alpha}\right\}$ are on the circle centered at the origin $O$ and of radius $\sqrt{\delta}$. Furthermore, let the arclength between any two centers be $c \delta^{3 / 4}$. We claim that we exactly need $N=c \delta^{-1 / 4}$ unit balls $B_{1}, \ldots, B_{N}$ such that

$$
\bigcup_{\alpha} B_{\alpha} \subset \bigcup_{i=1}^{N}(1+\delta) B_{i} .
$$

We consider two balls $B_{1}$ and $B_{2}$ centered at $O_{1}$ and $O_{2}$ respectively (as shown on Figure 5).

Let $O A \perp O_{1} O_{2}, O F \perp A O_{2}, A E=h, A O_{2}=A O_{1}=l$, and let $\angle A O F=\theta$, thus $\angle E O_{2} A=\theta$. Moreover, 


$$
\sin \theta=\sin \left(\angle E O_{2} A\right)=\frac{\overline{A E}}{\overline{A O_{2}}}=h / l
$$

also

$$
\sin \theta=\sin (\angle A O F)=\frac{\overline{A F}}{\overline{A O}}=\frac{l / 2}{\sqrt{\delta}} .
$$

Thus $\frac{l / 2}{\sqrt{\delta}}=h / l$, i.e.,

$$
l^{2}=2 \sqrt{\delta} h
$$

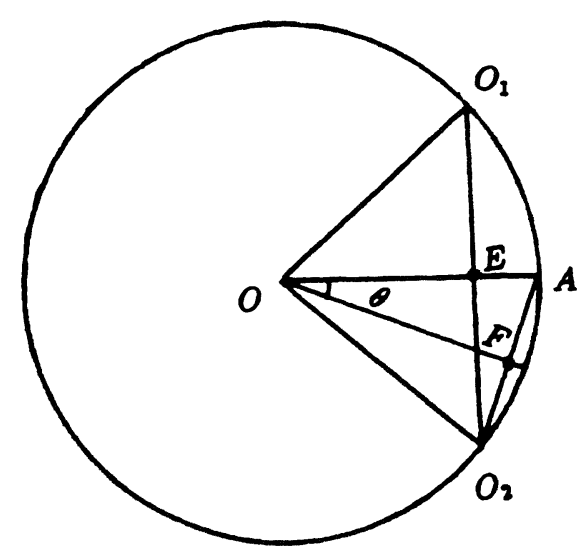

Figure 5

But by Proposition 4.3, $(1+\delta) B_{1} \cup(1+\delta) B_{2}$ can cover all unit balls $\left\{B_{\alpha}\right\}$ centered between $O_{1}$ and $O_{2}$ if and only if $h \leq c \delta$, i.e., $l \leq c \delta^{3 / 4}$. We also note that the arclength of $O_{1} O_{2} \leq c \delta^{3 / 4}$ if and only if $l \leq c \delta^{3 / 4}$. Hence, we exactly need $N=c \sqrt{\delta} / \delta^{3 / 4}=c \delta^{-1 / 4}$ balls.

\section{Proof of the Main Covering Lemma}

We now prove the main covering lemma (Lemma 1) stated in the introduction. We will need the following lemmas and propositions. 
Lemma 5.1. Let $\delta$ be small enough. Given any cube $Q$ in $\mathbf{R}^{2}$ with sidelength $2^{k} \sqrt{\delta}$ and let $\left\{B_{\alpha}\right\}_{\alpha \in \mathrm{I}}$ be any finite collection of balls with $r \leq \varrho\left(B_{\alpha}\right) \leq r+$ $2^{k} \delta$ for some $2^{k} \leq r \leq 2^{k+1}$ and centered in $Q$, where $k$ is an integer. Then one can select a subcollection of balls $B_{1}, \ldots, B_{N}$ such that

$$
\begin{gathered}
\bigcup_{\alpha} B_{\alpha} \subset \bigcup_{i=1}^{N}(1+c \delta) B_{i}, \\
N \leq c \delta^{-1 / 4} .
\end{gathered}
$$

The proof of Lemma 5.1 is straightforward if we use Lemma 4.1 and the scaling property.

Lemma 5.2. Let $\delta$ be small enough. Given any cube $Q$ in $\mathbf{R}^{2}$ with sidelength $2^{k} \sqrt{\delta}$ and let $\left\{B_{\alpha}\right\}_{\alpha \in \mathrm{I}}$ be any finite collection of balls with $2^{k} \leq \varrho\left(B_{\alpha}\right) \leq 2^{k+1}$. Then one can select a subcollection $B_{1}, \ldots, B_{N}$ such that

$$
\begin{gathered}
\bigcup_{\alpha} B_{\alpha} \subset \bigcup_{i=1}^{N}(1+c \delta) B_{i}, \\
N \leq c \delta^{-3 / 4} .
\end{gathered}
$$

PROOF. With no loss of generaliy, we may assume the largest ball $B$ in our collection is of radius $2^{k+1}$. Any other ball $B_{\alpha}$ with $\varrho\left(B_{\alpha}\right) \leq 2^{k+1}-2^{k} \sqrt{2 \delta}$ is contained in $B$. Thus the balls $B_{\alpha}$ with $\varrho\left(B_{\alpha}\right) \leq 2^{k+1}-2^{k} \sqrt{2 \delta}$ may be ignored. Now we partition the radii into the following intervals:

$$
2^{k+1}-2^{k} \sqrt{2 \delta}+2^{k} \delta l \leq \varrho\left(B_{\alpha}\right) \leq 2^{k+1}-2^{k} \sqrt{2 \delta}+2^{k} \delta(l+1)
$$

for $0 \leq l \leq \sigma$.

Thus $\sigma \approx \sqrt{2 / \delta}$. Applying Lemma 5.1 to these balls $\left\{\boldsymbol{B}_{\alpha}^{l}\right\}$ whose radii are in the interval corresponding to $l, 0 \leq l \leq \sigma$, we can select $\left\{\boldsymbol{B}_{i}^{l}\right\}_{i=1}^{N_{l}}$ such that

$$
\begin{gathered}
\bigcup_{\alpha} B_{\alpha}^{l} \subset \bigcup_{i=1}^{N_{l}}(1+c \delta) B_{i}^{l}, \\
N_{l} \leq c \delta^{-1 / 4} .
\end{gathered}
$$


Thus

$$
\bigcup_{\alpha} B_{\alpha} \subset \bigcup_{\alpha, l} B_{\alpha}^{l} \bigcup B \subset \bigcup_{i, l}(1+c \delta) B_{i}^{l} \bigcup B
$$

and the number of balls $\left\{\boldsymbol{B}_{\boldsymbol{i}}^{l}\right\}_{i, l}$ is less than

$$
\sum_{l=1}^{\sigma} N_{l} \leq \sigma \cdot c \delta^{-1 / 4} \leq c \delta^{-1 / 2} \delta^{-1 / 4}=c \delta^{-3 / 4}
$$

Therefore the subcollection $\left\{\boldsymbol{B}_{\boldsymbol{i}}\right\}$ will be the one in our lemma.

Now we can show the following:

Lemma 5.3. Let $\delta$ be small enough and $\left\{B_{\alpha}\right\}_{\alpha \in \mathrm{I}}$ be a finite collection of balls with $2^{k} \leq \varrho\left(B_{\alpha}\right) \leq 2^{k+1}$, where $k$ are integers. Then one can select balls $B_{1}, \ldots, B_{N}$ such that

$$
\bigcup_{\alpha} B_{\alpha} \subset \bigcup_{i=1}^{N}(1+c \delta) B_{i}
$$

and

$$
\sum_{i=1}^{N} \chi_{B_{i}}(x) \leq c \delta^{-7 / 4}
$$

for all $x \in \mathbf{R}^{2}$.

Proof. We subdivide $\mathbf{R}^{2}$ into a dyadic grid of cubes $\left\{Q_{j}\right\}_{j=1}^{\infty}$ whose sidelengths are all $2^{k} \sqrt{\delta}$. Let $\left\{\boldsymbol{B}_{\alpha}^{j}\right\}$ be the subcollection of $\left\{\boldsymbol{B}_{\alpha}\right\}$ with centers inside $Q_{j}$. For each $\left\{\boldsymbol{B}_{\alpha}^{j}\right\}$ and $Q_{j}$, we apply Lemma 5.2 to select $\left\{\boldsymbol{B}_{i}^{j_{i}}\right\}_{i=1}^{N_{j}}$ such that

$$
\begin{gathered}
\bigcup_{\alpha} B_{\alpha}^{j} \subset \bigcup_{i=1}^{N_{j}}(1+c \delta) B_{i}^{j}, \\
N_{j} \leq c \delta^{-3 / 4} .
\end{gathered}
$$

where $c$ is independent of $\delta, k, j$.

Then

$$
\bigcup_{\alpha} B_{\alpha} \subset \bigcup_{\alpha, j} B_{\alpha}^{j} \subset \bigcup_{j} \bigcup_{i=1}^{N_{j}}(1+c \delta) B_{i}^{j}=\bigcup_{i, j}(1+c \delta) B_{i}^{j}
$$


which shows (5.4). Now let $x_{0} \in \mathbf{R}^{2}$ be a fixed point and let $B\left(x_{0}, 2^{k+1}\right)$ denote the ball centered at $x_{0}$ and of radius $2^{k+1}$. Since $\varrho\left(B_{i}^{j}\right) \leq 2^{k+1}, \chi_{B_{i}^{j}}\left(x_{0}\right)$ vanishes for those balls $\left\{B_{i}^{i}\right\}$ centered outside $B\left(x_{0}, 2^{k+1}\right)$. Now the cardinality of $\{j\}$ such that $Q_{j} \cap B\left(x_{0}, 2^{k+1}\right) \neq \varnothing$ is no more than $c \delta^{-1}$. Thus

$$
\begin{aligned}
\sum_{i, j} \chi_{B_{i}^{j}}\left(x_{0}\right) & =\sum_{j} \sum_{i=1}^{\mathrm{N}_{j}} \chi_{B_{i}^{j}}\left(x_{0}\right) \\
& \leq \sum_{j} c \delta^{-3 / 4} \leq c \delta^{-1} \delta^{-3 / 4}=c \delta^{-7 / 4}
\end{aligned}
$$

this shows (5.5). Since $x_{0}$ is arbitrary, the proof is complete.

We now prove the main covering lemma.

We first select a ball $B_{1}$ with the largest radius. Having selected $B_{1}, \ldots$, $B_{k-1}$ select $B_{k}$ such that

$$
B_{k} \oplus \bigcup_{i=1}^{k-1}(1+\delta) B_{i}
$$

and $B_{k}$ has the largest possible radius out of the balls in the collection $\left\{B_{\alpha}\right\}_{\alpha \in I} \backslash\left\{B_{i}\right\}_{i=1}^{k-1}$. Thus clearly, we have

$$
\bigcup_{\alpha} B_{\alpha} \subset \bigcup_{i=1}^{N}(1+\delta) B_{i}
$$

Now let $B_{i}^{\prime}=(1+\delta) B_{i}$ and $\left\{B_{i, k}^{\prime}\right\}$ be the subcollection of $\left\{B_{i}^{\prime}\right\}_{i=1}^{N}$ such that $2^{k} \leq \varrho\left(B_{i, k}^{\prime}\right) \leq 2^{k+1}$. Then by using Lemma 5.3, we can select a subcollection $\left\{B_{i, k}^{\prime}\right\}$ of $\left\{B_{i, k}^{\prime}\right\}$ such that

$$
\begin{gathered}
\bigcup_{i} B_{i, k}^{\prime} \subset \bigcup_{j=1}^{N_{k}}(1+c \delta) B_{i j, k}^{\prime}, \\
\sum_{j=1}^{N_{k}} \chi_{B_{i, k}^{\prime}}(x) \leq c \delta^{-7 / 4},
\end{gathered}
$$

for all $x \in \mathbf{R}^{2}$, where $c$ is independent of $\delta, k$ and $k=0, \pm 1 \pm 2, \ldots$ Thus, we have 


$$
\begin{aligned}
\bigcup_{\alpha} B_{\alpha} & \subset \bigcup_{i=1}^{N}(1+\delta) B_{i} \\
& =\bigcup_{i=1}^{N} B_{i}^{\prime}=\bigcup_{k} \bigcup_{i} B_{i, k}^{\prime} \\
& \subset \bigcup_{k} \bigcup_{j=1}^{N_{k}}(1+c \delta) B_{i j, k}^{\prime} \\
& =\bigcup_{k, j}(1+c \delta)(1+\delta) B_{i j, k} \\
& \subset \bigcup_{j, k}(1+2 c \delta) B_{i j, k}
\end{aligned}
$$

for $c>2$ and $\delta$ small enough. Thus, the subcollection $\left\{B_{i j, k}\right\}$ satisfies (i) of Lemma 1.

Fix $x_{0} \in \mathbf{R}^{2}$. There exists $k_{0}=k\left(x_{0}\right)$ and $\sigma_{0}=\sigma\left(x_{0}\right)$ such that

$$
\begin{aligned}
\sum_{j, k} \chi_{B_{i, k}}\left(x_{0}\right) & =\sum_{k=k_{0}}^{k_{0}+\sigma_{0}} \sum_{j=1}^{N_{k}} \chi_{B_{i, k}}\left(x_{0}\right) \\
& \leq \sum_{k=k_{0}}^{k_{0}+\sigma_{0}}\left(\sum_{j=1}^{N_{k}} \chi_{B_{i, k}^{\prime}\left(x_{0}\right)}\right) \\
& \leq \sum_{k=k_{0}}^{k_{0}+\sigma_{0}} c \delta^{-7 / 4}=c \sigma_{0} \delta^{-7 / 4} .
\end{aligned}
$$

We claim $2^{\sigma_{0}} \leq 8 \delta^{-1}$. For otherwise there would exist a ball $B_{i_{j}, k_{0}+\sigma_{0}}$ for some $j$ with $x_{0} \in B_{i_{j}, k_{0}+\sigma_{0}}$ such that

$$
\varrho\left(B_{i j, k_{0}+\sigma_{0}}\right) \geq 1 / 2 \varrho\left(B_{i j, k+\sigma_{0}}^{\prime}\right) \geq 1 / 2 \cdot 2^{k_{0}+\sigma_{0}} \geq 2 \delta^{-1} 2^{k_{0}+1} .
$$

The first inequality above is due to the fact that

$$
\varrho\left(B_{i j, k_{0}+\sigma_{0}}^{\prime}\right)=(1+\delta) \varrho\left(B_{i j, k_{0}+\sigma_{0}}\right) \leq 2 \varrho\left(B_{i j, k_{0}+\sigma_{0}}\right) \text { for } \delta<1 .
$$

Let $x_{0} \in B_{i_{h}, k_{0}}$ for some $h$ be the ball with

$$
\varrho\left(B_{i_{h}, k_{0}}\right) \leq \varrho\left(B_{i_{h}, k_{0}}^{\prime}\right) \leq 2^{k_{0}+1} .
$$

Then $(1+\delta) B_{i_{j}, k_{0}+\sigma_{0}} \supset B_{i_{h}, k_{0}}$ which is a contradiction to (5.6). Thus $2^{\sigma_{0}} \leq 8 \delta^{-1}$, and the claim follows. That is, $\sigma_{0} \leq c \log \delta^{-1}$. Therefore,

$$
\sum_{j, k} \chi_{B_{i, k}}\left(x_{0}\right) \leq c \sigma_{0} \delta^{-7 / 4} \leq c \log \left(\delta^{-1}\right) \delta^{-7 / 4} .
$$

Since $x_{0}$ is arbitrary, we are done. 


\section{Proof of Theorem 1}

This section is devoted to the proof of the main theorem in this note (Theorem 1). We begin with recalling the following theorem in [DF2].

Theorem 6.1 (Donnelly-Fefferman). Let $M, u, \lambda$ be as in the introduction. Let $B(x, \delta)$ denote the ball centered at $x$ of radius $\delta$. Then

$$
\begin{gathered}
\int_{B\left(x, \delta\left(1+\lambda^{-1 / 2}\right)\right)}|u|^{2} \leq c \int_{B(x, \delta)}|u|^{2} \\
{\left[\int_{B(x, \delta)}|\nabla u|^{2}\right]^{1 / 2} \leq c \frac{\sqrt{\lambda}}{\delta}\left[\int_{B(x, \delta)}|u|^{2}\right]^{1 / 2}}
\end{gathered}
$$

Now we start the proof of Theorem 1 by showing the following lemmas.

Lemma 6.4. Let $u, \lambda$ as above, $1 \leq q<\infty$, then $u$ satisfies the Reverse-Hölder inequality

$$
\left[\frac{1}{|B|} \int_{B}|u|^{q}\right]^{1 / q} \leq c \sqrt{\lambda}\left[\frac{1}{|B|} \int_{B}|u|^{2}\right]^{1 / 2}
$$

where $c$ depends on $q$.

Proof. By the Poincaré inequality, for any ball $B$, we have

$$
\left[\frac{1}{|B|} \int_{B}\left|u-u_{B}\right|^{q}\right]^{1 / q} \leq c|B|^{1 / 2}\left[\frac{1}{|B|} \int_{B}|\nabla u|^{p}\right]^{1 / p}
$$

where $u_{B}=\frac{1}{|B|} \int_{B} u$ and $1<p<2,1 / q=1 / p-1 / 2$, and $c=c(p, q)$.

Applying Hölder inequality and (6.3) to the right side of (6.6), we obtain

$$
\left[\frac{1}{|B|} \int_{B}\left|u-u_{B}\right|^{q}\right]^{1 / q} \leq c \sqrt{\lambda}\left[\frac{1}{|B|} \int_{B}|u|^{2}\right]^{1 / 2} .
$$

By Minkowski's inequality, Lemma 6.4 follows for $2<q<\infty$, for the case $1<q \leq 2$, we can apply Hölder inequality again. 
Our theorem will follow from the following

Lemma 6.7. Suppose $w>0, q>2, \epsilon>0$ and $1+\epsilon \geq q^{\prime}$ where $1 / q^{\prime}=1-1 / q$, also assume that

$$
\int_{B\left(x, \delta\left(1+\lambda^{-1 / 2}\right)\right)} w \leq c_{0} \int_{B(x, \delta)} w
$$

and

$$
\left(\frac{1}{|B|} \int_{B} w^{q}\right)^{1 / q} \leq c_{1} \lambda \frac{1}{|B|} \int_{B} w
$$

then

$$
\|\log w\|_{B M O} \leq c \lambda^{15 / 8+\epsilon}
$$

where $c=c\left(c_{0}, c_{1}\right)$.

Theorem 1 will follow if we choose $w=|u|^{2}$.

In order to prove Lemma 6.7, we need the following

Lemma 6.9. Let $w, q, 0<\epsilon<1$ satisfy the hypothesis of Lemma 6.7, let $B$ be a fixed ball, $E \subset B$, then there exist $c_{2}, c_{3}$ such that if

$$
|E| \geq\left(1-c_{2} \lambda^{-15 / 8-\epsilon}(\log \lambda)^{-1}\right)^{k} \int_{B} w
$$

then

$$
\int_{E} w \geq\left(c_{3} \lambda^{-7 / 8}(\log \lambda)^{-1}\right)^{k} \int_{E} w
$$

where $c_{2}=c\left(c_{1}\right), c_{3}=c\left(c_{0}\right)$.

Proof. We proceed as the proof given in $[\mathrm{CM}]$. The method is to use induction on $k$. We first verify the lemma for $k=1$. To do so, we claim that if $\epsilon>0,|E| \geq\left(1-\bar{c} \lambda^{-(1+\epsilon)}\right)|B|$ for some appropriate $\bar{c}=\bar{c}\left(c_{1}\right)$, then

$$
\int_{E} w \geq 1 / 2 \int_{B} w
$$

To show this, we first note that $|B \backslash E| \leq \bar{c} \lambda^{-(1+\epsilon)}|B|$. If we choose $q>2$ such that $\frac{1+\epsilon}{q^{\prime}} \geq 1$, thus by (6.8), 


$$
\begin{aligned}
\int_{B \backslash E} w & \leq\left(\int_{B} w^{q}\right)^{1 / q}|B \backslash E|^{1 / q^{\prime}} \\
& \leq c_{1} \bar{c}^{1 / q^{\prime}} \lambda^{-\frac{1+\epsilon}{q^{\prime}}+1} \int_{B} w \\
& \leq c_{1} \bar{c}^{1 / q^{\prime}} \int_{B} w
\end{aligned}
$$

If we choose $\bar{c}$ such that $c_{1} \bar{c}^{1 / q^{\prime}}<1 / 2$, then $\int_{B \backslash E} w \leq 1 / 2 \int_{B} w$, this implies $\int_{E} w>\frac{1}{2} \int_{B} w$. Here we want to point out the choice of $\bar{c}$ is dependent on $\epsilon$ since $c_{1}=c_{1}(q)$ and $q$ is dependent on $\epsilon$.

Thus if $c_{2} \leq \bar{c}$, and $|E| \geq\left(1-c_{2} \lambda^{-15 / 8-\epsilon}(\log \lambda)^{-1}\right)|B|$, then

$$
\int_{E} w \geq 1 / 2 \int_{B} w \geq c_{3}\left(\lambda^{-7 / 8}(\log \lambda)^{-1}\right) \int_{B} w
$$

and we are done for the case $k=1$. Now we assume the statement is true for $k-1$. Obviously we can assume $|E| \leq\left(1-\bar{c} \lambda^{-(1+\epsilon)}\right)|B|$, otherwise, there is nothing to prove. Thus for each density point $x$ of $E$, we can select a ball $B_{x} \subset B$ such that $x \in B_{x}$, and

$$
\frac{\left|B_{x} \bigcap E\right|}{\left|B_{x}\right|}=1-\bar{c} \lambda^{-(1+\epsilon)}
$$

Applying Lemma 1 to the balls $B_{x}$ with the choice $\delta=\lambda^{-1 / 2}$, and with no loss of generality, assume $\left\{\boldsymbol{B}_{x}\right\}$ are finite, and define

$$
E_{1}=\left[\bigcup_{i=1}^{N}\left(1+\lambda^{-1 / 2}\right) B_{i}\right] \bigcap B
$$

Then $E_{1} \subset B$, and as the proof given in $[\mathrm{CM}]$, we can show

$$
\begin{gathered}
|E| \leq\left(1-c_{2} \lambda^{-15 / 8-\epsilon}(\log \lambda)^{-1}\right)\left|E_{1}\right| \\
\int_{E} w \geq c_{3} \lambda^{-7 / 8}(\log \lambda)^{-1} \int_{E_{1}} w
\end{gathered}
$$

for some $c_{2}=c\left(c_{1}\right), c_{3}=c\left(c_{0}\right)$. 
Now we prove Lemma 6.7. The proof will be almost the same as that given in $[\mathrm{CM}]$. In order to get the precise estimate, we like to show the details. It will be enough to assume $\frac{1}{|B|} \int_{B} w=1$. It is also sufficient to show

$$
\left|\left\{x \in B: w^{-1}(x)>t\right\}\right| \leq-\frac{|B|}{t^{c \lambda^{-1 / 5 / 8-}(\log \lambda)^{-2}}} .
$$

It is equivalent to show

$$
|\{x \in B: w(x)<t\}| \leq t^{c \lambda^{-15 / 8-t}(\log \lambda)^{-2}}|B| .
$$

Let us denote by $E=\{x \in B: w(x)<t\}$. Select $k_{0}$ such that

$$
|E| \approx\left[1-c_{2} \lambda^{-15 / 8-\epsilon}(\log \lambda)^{-1}\right]^{k_{0}}|B| .
$$

Thus

$$
k_{0} \approx c\left(\lambda^{15 / 8+\epsilon} \log \lambda\right) \log \left(\frac{|B|}{|E|}\right)
$$

Then by Lemma 6.9, and the normalization $\frac{1}{|B|} \int_{B} w=1$, we have

$$
\begin{aligned}
|B| & =\int_{B} w \leq\left(c_{3}^{-1} \lambda^{7 / 8} \log \lambda\right)^{k_{0}} \int_{E} w \\
& \leq\left(c_{3}^{-1} \lambda^{7 / 8} \log \lambda\right)^{k_{0}} t|E| .
\end{aligned}
$$

Thus

$$
\begin{aligned}
\frac{|B|}{|E|} & \leq t e^{k_{0} \log \left(c_{3}{ }^{1} \lambda^{7 / 8} \log \lambda\right)} \\
& \leq t\left(\frac{|B|}{|E|}\right)^{\left(c \lambda^{15 * \cdots} \log \lambda\right) \log \left(c_{3}^{-1} \lambda^{7 \cdot 8} \log \lambda\right)} \\
& \leq t\left(\frac{|B|}{\mid E}\right)^{c^{\prime} \lambda^{15 / 8 \cdots}(\log \lambda)^{2}}
\end{aligned}
$$


Hence

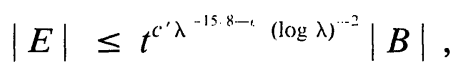

where $c^{\prime}$ is dependent on the constant $c$ in (6.4). Since $\epsilon$ is arbitrary, we can even have

$$
|E| \leq t^{c^{\prime \prime} \lambda^{-158-t}}|B| \text {. }
$$

Remark. Since for any $0<\epsilon<1$, we can choose $q$ so that $1+\epsilon \geq q^{\prime}$, and note that for such $q$, Lemma 6.7 holds. Unfortunately, the constant $c$ on the right side of (6.5) depends on $q$ (and then depends on $\epsilon$ ), and is unbounded when $q \rightarrow \infty$. Thus we have proved the Theorem 1 for any $\epsilon>0$. However we can not obtain the theorem 1 for replacing $\lambda^{15 / 8+c}$ by $\lambda^{15 / 8}$ since when $\epsilon \rightarrow 0$, $c_{\epsilon}$ is not bounded.

\title{
References
}

[B J. Brüning, Über Knoten von Eigfunktionen des Laplace Beltrami operator, Math. Z. 158, 15-21 (1978).

C) S. Y. Cheng, Eigenfunctions and nodal sets, Comment. Math. Helv. 51, 43-55 (1976).

[CM $]$ S. Chanillo and B. Muckenhoupt, Nodal Geometry on Riemannian Manifolds, J. Diff. Geom. 34(1), 1991.

CoW $\mid$ R. Coifman and G. Weiss, Analyse harmonique non-commutative sur certain espaces homogènes, Springer-Verlag, 1971.

[Do M. do Carmo, Differential geometry of curves and surfaces, Prentice-Hall, 1976.

[DF1] H. Donnelly and C. Fefferman, Nodal sets of eigenfunctions on Riemannian manifolds, Inv. Math. 93 (1988), 161-183.

[DF2 H. Donnelly and C. Fefferman, Growth and Geometry of eigenfunctions of the Laplacian Analysis and PDE, Lecture notes in Pure and Appl. Math., 122, Dekker, NY, 1990.

$[\mathrm{F}] \quad$ H. Federer, Geometric measure theory, Springer-Verlag, 1969.

L Covering Lemmas and an application to nodal geometry on Riemannian manifolds, preprint.

[SW $\mid$ E. T. Sawyer and R. L. Wheeden, Weighted inequalities for fractional and Poisson integrals in Euclidean and homogeneous spaces, to appear on Amer. Jour. of Math.

Recibido: 29 de Noviembre de 1990

\author{
Guozhen LU \\ Department of Mathematics \\ Rutgers University \\ New Brunswick, NJ 08903 \\ U.S.A.
}

Added in Proof: After this paper had been accepted for publication, the author has also shown a higher dimensional covering lemma in $\mathbf{R}^{\prime \prime}(n \geq 3)$ by which one can refine the BMI) norm estimate of $|\mathrm{CM}|$ by reducing the power of $\lambda$ to $n-1 / 8 \mid \mathrm{L}_{i}$. 